\title{
Elementos a considerar en la enseñanza del registro semiótico de las figuras en los primeros ciclos de la educación básica
}

Gustavo A. Marmolejo A. ${ }^{1}$

\section{Resumen}

La figura desempeña en el aprendizaje de la geometría un papel determinante. Pero, muchos estudiantes de forma espontánea no recurren a las posibilidades que brindan estas representaciones al intentar resolver problemas geométricos. En consecuencia, la enseñanza de las posibilidades que brinda este registro de representación es un asunto a considerar en la enseñanza de las matemáticas. En esta conferencia se pretende llamar la atención sobre la complejidad que subyace en la discriminación y designación de subfiguras y sub-configuraciones en una configuración geométrica.

Palabras claves: figuras, visualización y designación

\section{Summary}

You figure it it recovers in the learning of the geometry a determinant paper. But, many students of spontaneous form do not resort to the possibilities that offer these representations on having tried to solve geometric problems. In consequence, the education of the possibilities that offers this record of representation is a matter to considering in the education of the mathematics. In this conference it is tried to be call the attention on the complexity that sublies in the discrimination and designation of sub-figures and subconfigurations in a geometric configuration.

Keywords: figures, visualization and designation

\section{Introducción}

E n el aprendizaje de la geometría se recurre como mínimo a dos registros de representación semiótica: las figuras geométricas y el discurso teórico en lengua natural. En el primero se designan las figuras y sus propiedades; en el segundo, se enuncian los elementos que conforman el marco teórico sobre el cual se sustenta la clase de geometría sobre la cual se reflexiona. Para asegurar un aprendizaje de esta disciplina es necesario que los tratamientos propios a uno y otro registro se efectúen simultáneamente y de manera interactiva.

La originalidad de los procesos geométricos en relación a otros tipos de actividad matemática

\footnotetext{
${ }^{1}$ Doctorando en Educación Matemática. Universidad de Salamanca (España). Profesor del Departamento de Matemáticas y Estadística. Universidad de Nariño. usalgamav@mail.com
} 
tiene que ver con que es estrictamente necesaria la coordinación entre los tratamientos que provienen de estos dos registros. Pero, la mayoría de los estudiantes se encuentran muy lejos de alcanzar dicha coordinación, Duval [2] ha puesto en evidencia que uno de los mayores problemas en el aprendizaje de la geometría es que muy pocos alumnos logran la coordinación necesaria entre los tratamientos figurales y los tratamientos discursivos, incluso después de la educación básica y media.

Una de las razones que explica esta deficiencia tiene que ver con la naturaleza de estos registros, que no son exclusivos de las matemáticas. Los tratamientos figurales parecen proceder de leyes de organización de la percepción visual, y la práctica de un discurso teórico parece ser la prolongación directa de la comprensión inmediata de la lengua utilizada para comunicar [2].

En consecuencia, existe la creencia de que hay una proximidad entre los tratamientos que son naturales a cada uno de estos dos registros y aquellos que la actividad matemática solicita, en palabras de Duval este resulta ser un fenómeno de falsa proximidad.

Para lograr una coordinación entre los tratamientos propios a cada uno de los registros de representación enunciados arriba, se hace necesario como mínimo, y en una primera instancia, suscitar en los estudiantes el desarrollo de situaciones de aula que de forma separada posibiliten un aprendizaje explícito de los tratamientos pertinentes y potentes a cada uno de los dos registros.

Esta investigación se interesa por la discriminación de las dificultades y posibilidades que pueden enfrentar los estudiantes al desarrollar tareas cuyo propósito aluda a comprender las posibilidades de transformación y de designación que permiten las figuras. Aspectos, uno y otro, de importancia relevante para, en una primera instancia, poder considerar propuestas de aprendizaje encaminadas a coordinar las representaciones que provienen de los registros de la lengua natural y de las figuras. Así, pues, son dos los propósitos de esta investigación. Por un lado, describir la complejidad que subyace al pasar de una aprehensión perceptual que impone una configuración a discriminar en ella sub-figuras o sub-configuraciones determinadas y, por otro, resaltar la designación de las figuras geométrica como una actividad de complejidad considerable y susceptible de aprendizaje.

\section{Marco teórico}

La importancia de las figuras geométricas radica en el hecho de que forman un importante soporte intuitivo para el desarrollo de las actividades geométricas, es decir, dejan ver mucho más de lo que los enunciados dicen, permiten la ilustración de proposiciones, la exploración heurística de situaciones complejas, posibilitan "vistazos" sinópticos sobre ellas y verificaciones subjetivas. En fin, permiten en la resolución de un problema o en la búsqueda de una demostración, la conducta de abducción consistente en delimitar de entrada la clase de hipótesis o alternativas que han de considerarse.

Hablar del papel heurístico de las figuras alude a que es la conducta de abducción la que guía la deducción. Sin embargo, en el momento en que los alumnos deben desarrollar situaciones donde las figuras juegan este papel, se observa que ellos se encuentran bastante lejos de poder acceder a sus enormes posibilidades y en muchos casos, por el contrario, le asocian propiedades que no le corresponden o llegan incluso a no ver nada significativo en ellas. Para describir cuál puede ser el aporte heurístico de una figura en un problema de geometría, se debe distinguir el tipo de aprehensión susceptible de sugerir la solución del problema. Duval [1] ha mostrado que una misma figura puede dar lugar a aprehensiones de naturaleza diferente: Aprehensión perceptiva, aprehensión operatoria y aprehensión discursiva. Y que en algunos casos estas formas de discriminación se subordinan unas a las otras, 
se relacionan, y en otros se oponen. A continuación nos centraremos en estos tres tipos de aprehensión.

Aprehensión Perceptiva. En este nivel se reconocen, de manera automática e inmediata, las diferentes unidades figurales que son discernibles en una figura dada. Esta forma de aprehensión está ligada a las leyes gestálticas de organización de la percepción: cuando las unidades figurales de dimensión 2 están separadas, su reconocimiento no tiene ningún tipo de dificultad; pero no sucede lo mismo cuando se encuentran integradas en una configuración. Esto sucede por dos razones diferentes. En primer lugar, algunas unidades figurales de dimensión 2 predominan sobre otras unidades también de dimensión 2, de conformidad con la ley gestáltica de cierre.

En segundo lugar, una figura geométrica contiene, con frecuencia, más unidades figurales elementales que las requeridas para construirlas.

Aprehensión Operatoria. Las posibilidades de exploración heurística que permiten las figuras, se encuentran íntimamente relacionadas con la gama de modificaciones posibles que se pueden realizar sobre una figura. Una figura puede dividirse en diversas sub- figuras a partir de las cuales se puede transformar en otra de contorno global diferente. Las modificaciones que tienen estas características son modificaciones mereológicas [1], ponen en juego las relaciones existentes entre las partes y el todo. Cuando se agranda, disminuye o se deforma una figura, hablamos de modificación óptica, que transforma una figura en otra apelando a su imagen [1]. Por otro lado, también es posible desplazar o rotar tanto la figura de partida como las sub-figuras que la componen, en relación con la orientación del campo en el que se destaca. Cuando esto sucede, hablamos de una modificación posicional [1]. Así, pues, la aprehensión operatoria de las figuras es "una aprehensión centrada sobre las modificaciones posibles de una figura de partida y por consiguiente sobre las reorganizaciones perceptivas que estas modificaciones introducen" [1]. Por cada modificación existen varias operaciones cognitivas que no matemáticas, que brindan a las figuras su productividad heurística. "La productividad heurística de una figura, en un problema de geometría, hace relación a que haya una congruencia entre una de las operaciones y uno de los tratamientos matemáticos posibles del problema propuesto" [1].

Aprehensión discursiva. En palabras de Duval [1] es un tipo de aprehensión inseparable de una doble referencia, por un lado, a una red semántica de objetos matemáticos y, por otra, a una axiomática local. En este sentido, se dice que está indisociablemente ligada a las aserciones correspondientes del enunciado. Dicho de otro modo, la aprehensión discursiva de una figura privilegia exclusivamente el estatus que el enunciado concede a sus proposiciones. Por tanto, se debe tener en cuenta que las figuras por sí mismas no constituyen un registro de tratamiento autónomo, es decir, no basta con un simple reconocimiento perceptivo en ellas para asignarle propiedades particulares. Se pone así en evidencia una condición esencial del registro del registro semiótico de las representaciones figurales, "una figura representa una situación geométrica sólo en la medida en que la significación de ciertas unidades figurales y de algunas de sus relaciones, estén explícitamente fijadas de entrada" [2].

\section{Metodología}

En esta investigación es de naturaleza cualitativa, descriptiva, interpretativa. La captación y selección de los datos se realizó de forma mixta. Su interpretación consideró, el análisis funcional propuesto por Duval [2] en relación a la actividad cognitiva vinculada a los registros semióticos, en particular lo relacionado con las figuras geométricas. La población estuvo constituida por 30 estudiantes de grado cuarto de educación básica de dos instituciones educativas de la ciudad de Santiago de Cali (Colombia). Las actividades implementadas en la investigación fueron dos (Anexo 1). Los momentos a través de los cuales 
la población participante desarrolló las tareas propuestas y reflexiono sobre las posibilidades de sus maneras de proceder fueron los siguientes: trabajo individual, trabajo en pequeños grupos y puesta en común. Los instrumentos empleados en la recolección de los datos fueron las producciones escritas de los estudiantes al resolver las tareas propuestas, las discusiones realizadas al trabajar en pequeños grupos y en la socialización de las posibilidades que brinda cada manera de proceder. En casos puntuales, con el propósito de ampliar o precisar las descripciones de los estudiantes, fueron aplicadas entrevistas semi-estructuradas.

Son dos las interrogantes que nos planteamos en este estudio, a saber: ¿Cómo los alumnos vencen la aprehensión perceptual ante la solicitud explicita de discriminar sub-figuras o configuraciones especificas en una figura dada? ¿Cuáles dificultades presentan los estudiantes al intentar acompañar discursivamente lo que ven en una figura geométrica? Así, pues, fueron dos las tareas implementadas en la investigación, una para dar respuesta a la primera de las cuestiones, la otra para la segunda. De esta manera, se tuvo en cuenta dos categorías: paso de la aprehensión perceptual a la aprehensión operatoria interna y designación figural, una por cada pregunta y tarea propuesta. En lo que sigue se describe estas categorías y los elementos que, en las maneras de proceder de la población participante, les describen.

Paso de la aprehensión perceptual a la aprehensión operatoria interna: refiere en el proceso de resolución de las tareas propuestas al momento en que se pasa de concebir la configuración de partida de forma icónica a discriminar en ella las sub-figuras y subconfiguraciones en estudio.

Designación figural: considera las operaciones que la población participante introduce para designar a las figuras, sub-figuras y sub-configuraciones en estudio.

\section{Resultados y discusión}

En relación al paso de la aprehensión perceptual a la aprehensión operatoria interna: son 10 las sub-configuraciones a considerar en la primera actividad y 4 sub-figuras y 7 sub-configuraciones en la segunda. Para el total de la población no representó ninguna dificultad discriminar en la primera actividad las sub-configuraciones que representan a los 5 cuadrados de menor área y al cuadrado de mayor área. Caso contrario sucedió con las cuatro sub-configuraciones restantes. Más del 70\% de los estudiantes que intentaron resolver la actividad propuesta consideró que en la configuración representada había únicamente 6 cuadrados y solo iniciaron la búsqueda de los restantes en el momento en que el investigador indicó la existencia de un número mayor de cuadrados. El costo temporal que representó la búsqueda y discriminación de los cuatro últimos cuadrados, en relación a los primeros, fue bastante alto, se invirtió en esta parte de la tarea más del doble del tiempo dedicado a la discriminación de los primeros cuadrados.

En la otra actividad la discriminación de las subfiguras que representan triángulos no representó ningún tipo de dificultad, todos los estudiantes dieron cuenta de ellos rápidamente. Lo contrario sucedió en la discriminación de los paralelogramos BCDF y el trapecio BDEA. Un total de 12 de los 30 estudiantes les fue imposible discriminarlos. Además, los que sí lo lograron invirtieron mayor tiempo en su búsqueda. Las parejas de trapecios y paralelogramos restantes, fueron discriminados solo 5 de los 30 estudiantes.

Estos resultados ponen de manifiesto que al discriminar sub-figuras y sub-configuraciones en una configuración dada es un asunto complejo. Son dos los factores de visibilidad presentes en la actividad que explican tal complejidad: el solapamiento de unas figuras en otras y la orientación de una representaciones en relación a otras. Así, pues, es indispensable poner en acto, y de manera previa a la discriminación 
de sub-figuras y sub-configuraciones en una configuración dada, actividades que susciten no solo la representación de figuras básicas en posiciones diferentes a la que habitualmente se acostumbra a representar; sino que además bajo la aplicación de operaciones (sean de rotación o de traslación) que los estudiantes puedan verificar la isometrías existente entre la figura inicial y la imagen que por rotación o traslación se han representado, de esta manera dejar de lado la diferencia perceptual que entre unas y otras se presenta.

En relación a la designación figural: si bien el hallazgo de algunas sub-configuraciones en las dos actividades propuestas representó un nivel de complejidad alto, este no se compara con el vivenciado por toda la población a la hora de designar, de forma clara y precisa, las sub-figuras y sub- configuraciones que habían encontrado. En un primer momento, 28 de 30 estudiantes manifestaron no comprender a qué hacía referencia tanto la consigna de la actividad como el investigador al exigirles que de forma escrita dieran a conocer cuáles fueron las figuras que habían encontrado en la configuración dada o se encontraban en situación de penuria nominum, es decir, en ausencia de palabras correspondientes disponibles [3]. Solo para 2 estudiantes la designación no representó tal complejidad. Ambos designaron las partes en estudio a través de la operación de descripción, por ejemplo, uno de ellos introdujo tres estrategias distintas. La primera, bajo el recurso de un demarcado uso de adverbios de posición, designó cada una de las figuras halladas a partir del lugar en el cual se ubicaban dentro de la configuración dada. La segunda, tuvo en cuenta que algunas de las sub-configuraciones no son representadas en la posición que habitualmente se acostumbra en textos escolares y en el aula de clase, de esta manera le describe a partir de su "parecido" con otras figuras (rombos). Por última, estableció algunas sub- configuraciones a partir del tipo y del número de sub-figuras que le conforma.
A partir de la insistencia del investigador para que realizaran la tarea propuesta, 27 de los 28 estudiantes que al encontrarse en situación de penuria nominum no habían podido iniciar una organización discursiva que dé cuenta de cuáles fueron las figuras encontradas, intentaron mediante uso de deícticos dar respuesta a la pregunta ¿Cuáles cuadrados? Es decir, señalaban con sus dedos o con la punta de un lapicero un punto de la superficie del cuadrado o el contorno del cuadrado al cual querían referirse y acompañan esta acción mediante la explicitación de los deícticos: este, ese, aquel, el que está allá, éste de acá. Pero al ser cuestionados por el investigador sobre la utilidad y precisión de esta forma de designación a la hora de ser llevada a la hoja de papel, todos coincidieron que no era la manera adecuada de designar las figuras, pues, quien leyese su escrito no podría dar cuenta de cuáles son los cuadrados a los que se refiere el escritor en cada momento. En otras palabras, se dieron cuenta que una designación pura de las figuras encontradas mediante la aplicación de deícticos basta para identificar de forma más o menos precisa el "objeto" del cual se habla dentro de un contexto de comunicación oral, pero nunca en el de una expresión escrita.

El resto de la población que inicialmente no le fue posible proponer una organización discursiva que dé cuenta de las figuras discriminadas (1 estudiante) introdujo una nueva estrategia. Discriminó algunas de las sub-configuraciones a partir de relación de equivalencia existente entre sus áreas. Así referencia unos y otros en los términos siguientes: "Yo encontré 7 cuadrados...5 pequeños y dos grandes..."

En breve, los anteriores resultados ponen en evidencia que la designación de figuras geométricas es una cuestión que genera complejidad alta en los estudiantes de los primeros cursos de la educación básica, en consecuencia, igual que en el paso de la aprehensión perceptual a la aprehensión operatoria interna, es un elemento a considerar en la enseñanza de la geometría. 


\section{Conclusiones}

La enseñanza de los tratamientos que proceden de los registros semióticos de las figuras y de la lengua natural es un asunto a considerar para asegurar la coordinación entre las representaciones que proceden de uno y otro registro. El desarrollo de investigaciones que describan las dificultades y posibilidades que brinda la aplicación de secuencias de enseñanza, cuyo propósito sea reflexionar sobre uno y otro aspecto es un elemento de gran importancia para comprender muchos de los fenómenos que subyacen al aprendizaje de la geometría. Esta investigación pone en evidencia que la designación figural y el paso de una aprehensión perceptual de una figura a discriminar en ella elementos bidimensionales internos, son aspectos que están lejos de ser obvios y espontáneos para la mayoría de los estudiantes. Así, pues, es necesario realizar estudios de mayor amplitud que permitan discriminar los mejores caminos para enfrentar y sobrepasar las dificultades evidenciadas en tales procesos.

\section{Bibliografía}

[1] Duval, R. (1998). Pour une approche cognitive des problèmes de géométrie en termes de congruence. Annales de Didactique et de Sciences Cognitives, 1, pp. 57-74

[2] Duval, R. (1999). Semiosis y Pensamiento humano. Registros semióticos y aprendizajes intelectuales. Santiago de Cali: Artes Graficas Univalle.

[3] Eco, U. Sémiotique et philosophie du langane. Paris: P.U.F. 1988. 